\title{
Analysis on the Burning Accident of 10kV Voltage Transformer
}

\author{
Si Yadong ${ }^{1}$, Bai Xue ${ }^{2}$, Ma Wei ${ }^{3}$, Zhang Yuhui ${ }^{3}$, Guan Ling ${ }^{4}$, Wang Xue ${ }^{4}$, Zhao Chuanzong ${ }^{4}$, \\ Liu Peng ${ }^{4}$, Liu Ren ${ }^{4}$, Tang Hui ${ }^{4}$ \\ ${ }^{1}$ State Grid Liaoning Electric Power Co., Ltd. Chaoyang power supply company. \\ ${ }^{2}$ State Grid Liaoning Electric Power Co., Ltd. Huludao power supply company. \\ ${ }^{3}$ State Grid Liaoning Electric Power Co., Ltd. Liaoyang power supply company. \\ ${ }^{4}$ State Grid Liaoning Electric Power Co., Ltd. Fushun power supply company
}

Keywords: Voltage transformer, Accident analysis, Preventive measures, Field test

\begin{abstract}
Voltage transformer (PT) is an instrument transformer, which is used to transform voltage on line. Chaoyang power supply company has occurred two $10 \mathrm{kV}$ three-phase five column voltage transformer burnt out accident. From the angle of accident analysis, the cause of the accident is analyzed. The main reason is the ferromagnetic resonance overvoltage. Based on the accident analysis and theoretical analysis and test, precautions and precautions to avoid similar accidents are put forward.
\end{abstract}

\section{Introduction}

The voltage transformer (PT) is an instrument transformer. An instrument for converting voltage on a line. It is mainly used to measure the voltage, power and electric energy of the circuit. It is used to protect the equipment in the circuit when the line breaks down, so the capacity of the voltage transformer is very small (only tens of volt ampere).

In Beipiao power supply company, two $10 \mathrm{kV}$ integrated A mobile substations adopt neutral point ungrounded mode. The electromagnetic three-phase five column voltage transformer is adopted, and the product model is "JSZW-10". Since the two $10 \mathrm{kV}$ substations have been running, voltage transformer burning accidents have occurred. According to accident investigation and analysis, they are all caused by ferromagnetic resonance overvoltage. In order to monitor the earthling alarm signal in time. Normally, the first side of the three coil voltage transformer is connected to a star circuit, and the neutral point is grounded. The second side circuit is a star circuit, and the neutral point is grounded. The third side circuit is the auxiliary coil, which is an open delta circuit. The neutral point of such a system is unstable, and it can give the real ground fault signal. However, ferroresonance will occur when the earth capacitance reactance and the ratio of excitation reactance to a certain value are saturated. The overvoltage also produces a fault signal. At the same time, due to the poor volt ampere characteristic of this type of voltage transformer, when ferromagnetic resonance occurs, the three-phase current of the voltage transformer will reach several times or even one hundred times the excitation current. At this time, it is very easy to cause the overheat accident of the coil of the voltage transformer.

\section{Cause analysis}

\subsection{The influence of volt ampere characteristic of voltage transformer}

Two typical volt ampere characteristics of core inductors have been simulated. The test results show that the better the volt ampere characteristics of the core inductor, that is, the more slow the core saturation is, the more the resonance area moves to the right, the greater the impedance parameter $\mathrm{XC0} / \mathrm{XL}$ is needed for the resonance. Conversely, the more shift to the left, the smaller the $\mathrm{XCO} / \mathrm{XL}$ required for resonance. In the fundamental resonant region, the impedance ratio $\mathrm{XC} / \mathrm{XL}$ is about 0.08 to 0.8 . In the high frequency resonant region, the impedance ratio $\mathrm{XC} 0 / \mathrm{XL}$ is 
about 0.6 to 3 . When the zero sequence capacitance of power grid is changed, the XC0/XL will change. In the loop, a resonance state may change to another resonance state. If the zero sequence capacitance is too large or too small, it can be divorced from the resonance region. Considering the fact that the actual situation of the voltage transformer is different from that of the simulation, the simulation test results are only used to qualitatively estimate the matching of the system impedance parameters. Because the type, date and manufacturer of voltage transformer are various, the resonant regions of voltage transformers should be determined according to actual tests.

At the scene, the capacitance current of the grid to the ground can be measured. According to $\mathrm{XC0/XL}$, estimate whether the power grid is in resonance zone. The capacitance current of the grid can also be calculated by the formula Ic $=3 U x C U$. In the formula, the lc- capacitance current, the unit "A"; the Ux- grid operation phase voltage, the unit " $k \mathrm{~V}$ "; the C- line to ground capacitance, the unit "Q". When $\mathrm{X0} / \mathrm{Xm}<0$. 01, no resonance occurs. With the increase of $(\mathrm{X} 0 / \mathrm{Xm}), 1 / 2$ frequency division, fundamental frequency and three frequency doubling resonance occur successively. Correspondingly, the applied voltage of resonance is also increasing. The operating voltage is usually rated phase voltage (0.58Ur, Ur is rated line voltage). In the $1 / 2$ frequency division, the fundamental resonance occurs more frequently, and the resonance of the high order harmonic is less. The frequency of frequency division resonance is not strictly equal to $1 / 2$ times. When the frequency division is resonant, the core is highly saturated, the excitation current increases dramatically, and can even increase to one hundred times the rated current, causing the voltage transformer to burn down or to protect the fuse to fuse.

\subsection{The influence of the structure of voltage transformer}

The three single-phase small capacity test transformers used in the field simulation test are equivalent to three single-phase voltage transformers. There are three single-phase voltage transformer groups and three phase five column voltage transformers on site. They are different in the resonance excitation. The experimental research shows that the starting voltage of single-phase voltage transformer group is lower than that of three-phase five column voltage transformer. The single phase voltage transformer unit can excite the resonance easily. This is mainly due to the difference in the structure of collision between them, resulting in the difference of zero sequence impedance.

The magnetic circuit of three core five column mutual inductor and single phase mutual inductor group. The magnetic circuit of the zero sequence flux of the single-phase transformer unit is the same as the magnetic circuit of the positive sequence flux, and each phase has its own closed loop. Therefore, the zero sequence impedance is equal to the positive sequence impedance. For the three core five column voltage transformer, because the zero sequence flux passes through two sides, the magnetic path is long, while the three core five column voltage transformer core section is small. Therefore, the zero sequence magnetic flux reluctance is much larger than that of the single-phase transformer group. As mentioned above, resonance is caused by zero sequence magnetic flux. The zero sequence flux of the three core five column transformer is large and the resonance is not easy to generate.

Due to the difference of magnetic circuit, the voltage used for calculating and testing zero sequence impedance of these two types of voltage transformers is different. The voltage acting on the voltage transformer is the superposition of positive sequence voltage and zero sequence resonance voltage when the power grid is resonant. For three core five column transformers, zero sequence voltage is close to phase voltage. Positive sequence voltage has little effect on zero sequence voltage impedance, so the corresponding inductance value under phase voltage is adopted.

\section{Preventive measures}

The analysis of ferromagnetic resonance is carried out based on the above field tests. To prevent ferromagnetic resonance, we need to reduce the scope of the resonance region as far as possible, or try to avoid the resonance region. It can be carried out from the following aspects:

1) When the ferroresonance occurs, the open delta circuit is temporarily shorted by relay 
protection. At this time, a certain part of the unstable region may be stabilized. But after the resonance, the short circuited open delta circuit should be opened immediately to avoid the abnormal indication signal when the system appears single-phase grounding.

2) The zero sequence capacitance Xco of the distribution system and the excitation reactance Xm of the voltage transformer are measured. If the two are equal, then a small capacity capacitor with a star neutral point will be connected to the distribution system. It is also possible to put a feeder cable on the two side of the bus before the substation is subjected to electricity, so that the ratio of the system to the reactance of the ground and the transformer is far away from the unstable region.

3) Reduce the operating voltage of the voltage transformer. In the $10 \mathrm{kV}$ distribution system, the operating voltage of the transformer is reduced from $10 \mathrm{kV}$ to $5.8 \mathrm{kV}$. The unstable region will be greatly reduced. The frequency division area will be reduced by half. The base frequency region has only a very small part. There is no unstable region with three harmonic waves. The disadvantage of this measure is that the voltage transformer does not connect the voltmeter outlet.

4) On the basis of inherent resistance of voltage transformer coil, additional external DC resistance is added. Narrowing the range of ferroresonance. When the resistance is connected, the capacity of the resistance should be clearly defined. Because the ferroresonance condition of voltage transformer is related to the saturation point of the transformer characteristic curve. In order to avoid voltage saturation due to voltage rise, the capacity of external DC resistance is best equal to the capacity of voltage transformer.

5) The methods of access resistance are:

(1) The neutral point of the primary side of the voltage transformer is grounded through the transition resistance. This measure can not only restrict the current in the voltage transformer, especially limit the high amplitude current flowing through the voltage transformer when interrupted arc grounding. It can also reduce the voltage on each phase voltage transformer (equivalent to improving the volt ampere characteristic of voltage transformer). The rated power of resistors must be large. Generally, nonlinear resistors with large rated power are connected in series with linear resistors. The nonlinear resistor has a greater resistance at low voltage and can prevent the development of resonance. For the serious asymmetry of the three-phase capacitor or the abnormal firing of the primary phase fuse of the voltage transformer, the harmonic elimination can be effectively eliminated. In general, the resistance value is $<0.15 \mathrm{Xm}$ ohm.

(2) The voltage transformer opens the triangle circuit and connects the resistance RA. R $\triangle$ is equivalent to the neutral point of the power transformer, so the smaller the resistance RA, the more restraining the resonance. If $\mathrm{RA}=0$, the two ends of the triangle circuit will be short circuited, equivalent to the direct grounding of the neutral point of the power grid, the resonance will not occur. In general, the resistance values are selected according to the $\mathrm{R} 0.45 \mathrm{Xm} / \mathrm{K} 13 * \mathrm{~K} 13$ formula, where $\mathrm{K} 13$ is the voltage ratio of the primary side and the open delta circuit of the voltage transformer. [1]

\section{Conclusions}

Ferromagnetic resonance has a great harm to voltage transformer. In order to avoid the occurrence of ferroresonance, through theoretical analysis and a large number of simulation experiments in the field, it is concluded that all the voltage transformers are in parallel with 50 to $60 \mathrm{ohm}$ adjustable resistance in the opening triangle. The effect is very obvious. After the improvement of the voltage transformer, no accident of voltage transformer burning has occurred, and good results have been achieved.

\section{References}

[1] Zhou Heping. Measures for eliminating ferroresonance of voltage transformer in neutral point insulation system. China electric power.1993.

[2] Li Linchuan. Electrical energy production process [M]. Science Press.2011. 
[3] Li Chao. Elementary introduction of voltage transformer. China Electric Power Publishing House.2013.

[4] Li Jian. Q \& A of substation maintenance and maintenance technology. China Power Publishing House, 2014.

[5] Zhang Hua, Zhu Tao and so on. Practical technology questions and answers for substation equipment operation. China Electric Power Publishing House.2013. 